\title{
Depression in African women presenting for psychological services at a general hospital
}

\author{
M Ngcobo, BJ Pillay \\ Department of Behavioural Medicine, Nelson R Mandela School of Medicine, Faculty of Health Sciences, \\ University of KwaZulu-Natal, South Africa
}

\begin{abstract}
Objective: Despite the increase in knowledge of depression, little is known about depression among African populations, especially African woman. In South Africa, inadequate mental health services generally and specifically for African people in our society, has led to under reporting and under diagnosing of the disorder. The object of the study was to understand depression in African women attending a state health service. Method: Clinical records of all patients presenting with depression to a general hospital located in a densely populated African township, over a 2 year period, were examined. Results: The epidemiological data is described. Depression in these women was related to poverty, overcrowding, unemployment, high levels of crime, lack of services and sexual abuse. Conclusion: African women return to a social context within which depression is increasingly prevalent. Establishing psychological services relevant to needs as well as means of ensuring that therapeutic gains extend to their social context are considered.
\end{abstract}

Keywords: Depression, Health in African women, Cross-cultural health, Mental health

Received: 26-01-2007

Accepted: 18-10-2007

\section{Introduction}

Women across cultures and around the world have an increased chance of suffering from depression compared to men. ${ }^{1}$ The ratio of depression in women to men is $2: 1$ and has been found to affect one in five women. ${ }^{2}$ Depression in women, world wide, seems to manifest mostly as physical complaints. ${ }^{3}$ There is a perception that this may be more so in African woman. Few depressed women actually go to a mental health practitioner when symptoms first manifest. They usually go to a general practitioner complaining of physical symptoms. ${ }^{1}$ This may contribute to health care professionals misdiagnosing depression in many of those seeking help, even when their symptoms are severe. The stigma associated with consulting a mental health practitioner may contribute to the general practitioner being preferred.

Research on gender differences in depression has progressed steadily in the last 20 years suggesting that women and men have the same causes of depression, such as, biological factors, personality characteristics, social conditions, and developmental

\section{Correspondence:}

Prof BJ Pillay

Department of Behavioural Medicine, Private Bag 7, Nelson R Mandela

School of Medicine, Faculty of Health Sciences,

University of Kwazulu Natal, Congella, Durban, 4013, South Africa

email: pillayb@ukzn.ac.za characteristics. ${ }^{4}$ However women have been found to be more susceptible to other factors such as neuroticism, substance use and early adversity. ${ }^{2,4}$

It has also been suggested that 'humiliating experiences', those of defeat and entrapment are at the core of depressive episodes in females even though females were far more unlikely to report such experiences due to their apparent social circumstances. ${ }^{5}$ It is further suggested that women may also tend to experience more personal change and a greater number of life events when compared to men. It is more often than not that these events are largely negative and have serious consequences on health status and behaviour. ${ }^{5}$

The severity of depression may also be greater in women than men. ${ }^{6}$ Guilt feelings are more pronounced, anxiety disorders more apparent and suicidal behaviour were encountered more frequently in women. ' 'Marriage' is considered a risk factor for woman in the development of depression. This is due to genderspecific demands placed on females by marriage and the limited 'other' roles for women (e.g. reduced possibility of being employed and increase of stress if employed. ${ }^{2}$ Childhood sexual abuse is also regarded a risk factor for depression in women. ${ }^{8}$ Unemployment, low income and low education are found to be related to depression. Since the majority of South Africans are struggling with these social issues, it can be implied that they may be more susceptible to depression. ${ }^{4}$ 
Finally understanding the significance of depression in African women requires the recognition of the fundamental importance of religion, culture and the African world view. ${ }^{9}$ A misdiagnosis of depression in black women may be due to the use of different terminology. A single term in isiZulu may refer to different states; some terms have a more complex linguistic structure in isiZulu than in English; while others have no equivalent meaning related to the specific emotion. While English contains a variety of terms referring to different symptoms and emotional states, in many instances, isiZulu equivalents do not exist. For example, "feeling blue" in the English language is associated with certain emotional states, this is not the case in isizulu and such a concept is meaningless if literally translated. On the other hand "kubuhlungu inhliziyo" is a popular isiZulu idiom referring to emotional pain or being disappointed. When literally translated it means 'I have pain in my heart'.

This study therefore looks at gender differences in depression as a result of social stress/impoverishment, presenting as psychosomatic illness and the appropriateness of the psychological services offered.

\section{Method}

A retrospective survey of the clinical records, of all patients presenting with depression or related symptoms to a clinical psychologists over a 2 year period, was undertaken. All patients were referred to the clinical psychologist after they were medically screened by a medical practitioner or psychiatry registrar/ psychiatrist. The sampled files were studied for demographic details such as age, gender, marital status, education, occupation and residential area, as well as: referral source; aetiology; psychosocial stressors; clinical features; diagnosis; availability of support systems; assessments done as well as number of sessions. Progress notes were also consulted for management information.

A total of 87 clinical files were identified and audited for the period of review. Files with information missing were excluded. Patients were assessed using a semi-structured clinical interview and mental status examination (MSE). Permission was obtained form the Hospital Manager to conduct the study.

\section{Results}

\section{Demographic distribution}

A total of 54 clinical files were analyzed. The patients' ages ranged from $19-64$ years. Fifty one (94\%) of the sample was black African, 2 (4\%) Indian and 1 (2\%) White. Amongst these, 10 (18.50\%) were males with a mean age of 30.10 (SD 13.07) years and 44 (81.50\%) females - mean age of 34.82 years (SD 1 1.78). There were no significant difference in the ages of the gender groups ( $t=1.121$, df 52; $p=0.268)$. Most patients were unemployed $41(75.9 \%)$ and amongst those who were employed, only 4 (7.4\%) had professional qualifications (i.e. teaching diploma and nursing diploma). Thirteen (24\%) had no formal education, 9 (16.6\%) had an education ranging from grade 1 - grade 7 and 36 (66.6\%) had an education ranging from grade 8 - grade 12 . There were no significant differences in the education status between the gender groups $(t=0.513$, df $52 ; p=0.610)$. With regards to marital status, 37 (68.5\%) were single, 9 (16.6\%) were married, 4 (7.4\%) divorced and $7.4 \%$ widowed, respectively. The majority of the patients were from the township; considerably fewer patients were from the immediate surrounding rural areas. Table I provides a demographic summary.

\begin{tabular}{|c|c|c|c|}
\hline \multicolumn{4}{|c|}{ Table I: Demographic distribution ( $N=54$ ) } \\
\hline & & $n$ & $\%$ \\
\hline Gender & $\begin{array}{l}\text { Male } \\
\text { Female }\end{array}$ & $\begin{array}{l}10 \\
41\end{array}$ & $\begin{array}{l}18.5 \\
75.9\end{array}$ \\
\hline Marital status & $\begin{array}{l}\text { Married } \\
\text { Single } \\
\text { Divorced } \\
\text { Widowed }\end{array}$ & $\begin{array}{c}9 \\
37 \\
4 \\
4\end{array}$ & $\begin{array}{r}16.6 \\
68.5 \\
7.4 \\
7.4\end{array}$ \\
\hline Education & $\begin{array}{l}\text { Grade 1-7 } \\
\text { Grade 8-12 } \\
\text { None }\end{array}$ & $\begin{array}{c}9 \\
36 \\
13\end{array}$ & $\begin{array}{l}16.6 \\
66.6 \\
24.0\end{array}$ \\
\hline Employment status & $\begin{array}{l}\text { Unemployed } \\
\text { Machinist } \\
\text { Cleaner } \\
\text { Nurse } \\
\text { Teacher } \\
\text { HIV Counsellor } \\
\text { Admin clerk } \\
\text { Driver }\end{array}$ & $\begin{array}{c}41 \\
2 \\
2 \\
2 \\
2 \\
1 \\
1 \\
1\end{array}$ & $\begin{array}{l}75.9 \\
3.7 \\
3.7 \\
3.7 \\
3.7 \\
1.8 \\
1.8 \\
1.8\end{array}$ \\
\hline
\end{tabular}

\section{Aetiology}

Forty eight (88.8\%) patients, reported an identifiable stressor/s for the cause of their depression, those with no formal education or limited formal education were more likely to cite a cultural cause, such as bewitchment (5.5\%), failure to perform some traditional ritual (3.7\%) or neglecting the call to be a diviner (1.8\%) - see Table II.

\section{Diagnosis}

The Diagnostic and Statistical Manual of Mental Disorders - IV TR (DSM-IV TR) nomenclature was employed. Results are presented in Table II. Major Depressive Disorder was diagnosed in 23 (42.5\%) patients, with 16.6\% being diagnosed with dysthymia, 9.2\% conversion disorder, 3.7\% psychosomatic disorder, and 5.5\% major depressive disorder with psychotic symptoms.

\begin{tabular}{|l|l|c|c|}
\hline \multicolumn{2}{|l|}{ Table II: Aetiology, Diagnosis and Social Support } \\
\hline \multirow{5}{*}{ Aetiology } & & $n$ & $\%$ \\
\hline \multirow{5}{*}{ Diagnosis } & Stress & 48 & 88.8 \\
& Bewitchment & 3 & 5.5 \\
& Role of the ancestors & 2 & 3.7 \\
& Spiritual & 1 & 1.8 \\
\hline \multirow{2}{*}{ Support structures } & MDD & 23 & 42.5 \\
& Dysthymia & 9 & 16.6 \\
& Conversion D/O & 5 & 9.2 \\
& Psychosomatic D/O & 2 & 3.7 \\
& MDD with psychosis & 3 & 5.5 \\
& None & 38 & 70.3 \\
& Mother & 5 & 9.2 \\
& Husband & 5 & 9.2 \\
& Sister & 3 & 5.5 \\
& Dad & 2 & 3.7 \\
& Church & 1 & 1.8 \\
\hline
\end{tabular}




\section{Support}

The majority of the patients, 38 (70.3\%), indicated a lack of actual and/ or perceived support. Five (9.2\%) cited their mother as their source of support, 5 (9.2\%) identified husbands as being supportive, 3 (5.5\%) indicated their sisters as supportive, 2 (3.7\%) identified their father as the support and l (1.8\%) received support from the church.

\section{Psychosocial factors}

The most common psychosocial stressor cited by most patients, 15 (27\%), was domestic violence and infidelity (mainly their husband's unfaithfulness with a family member). This was followed by unemployment, 13 (24\%), sexual abuse/ rape, 10 (18.5\%), relationship problems, 9 (16.6\%), being either attacked, mugged, and or having had their houses broken into 8 (15\%). Table III lists the other stressors.

\begin{tabular}{|l|c|c|}
\hline \multicolumn{2}{|l|}{ Table III: Psychosocial Stressors } \\
\hline Stressor & N & $\%$ \\
\hline Domestic violence \& infidelity & 15 & 27.0 \\
Unemployment & 13 & 24.0 \\
Sexual abuse/rape & 10 & 18.5 \\
Relationship problems & 9 & 16.6 \\
Mugging, break-ins, gunshot & 8 & 14.8 \\
Death & 6 & 11.1 \\
Homelessness & 3 & 5.5 \\
HIV status & 3 & 5.5 \\
Political violence & 3 & 5.5 \\
Termination of pregnancy & 2 & 3.7 \\
\hline
\end{tabular}

\section{Symptom presentation}

A significant percentage of the patients mentioned pain as one of the most troubling symptom, 4l(75.9\%). These included headaches, pain in the neck, backache, generalized body pains, and 'crippling' pain in the groin. Suicidal thoughts were prominent in $66.6 \%$ of the patients, and $18.5 \%$ had at least one suicide attempt. Insomnia was cited by $46 \%$ of the patients. See Table IV for a complete list of symptoms presented.

\section{Assessment, psychological intervention and adherence to therapy}

Since all patients referred to psychologists had experienced a crisis at the time of referral, following the initial psychological

\begin{tabular}{|l|c|c|}
\hline \multicolumn{2}{|l|}{ Table IV: Symptom presentation } \\
\hline Symptom reported & Total number & Percentage (\%) \\
\hline Pain & 41 & 75.9 \\
Suicidal ideation & 36 & 66.6 \\
Insomnia & 25 & 46 \\
Depressed mood & 14 & 25.9 \\
Suicide attempts & 10 & 18.5 \\
Tiredness & 5 & 9.2 \\
Tearfulness & 5 & 9.2 \\
Fainting spells & 2 & 3.7 \\
Poor performance & 2 & 3.7 \\
Hopelessness & 1 & 1.8 \\
Guilt & 1 & 1.8 \\
Hallucinations & 1 & 1.8 \\
\hline
\end{tabular}

assessment, crisis intervention took place . Few patients kept follow up sessions. Ideally weekly appointments for Cognitive -

Behaviour Therapy would have been prescribed. When possible, follow up appointments had to be scheduled at monthly intervals at the request of most patients so as to accommodate their financial circumstances. Most patients defaulted after one session. Thirty one (22.2\%) discontinued therapy after two sessions, 9.2\% discontinued after three sessions. One patient had four sessions of therapy, 1 had six sessions of therapy and only one continued for 12 sessions of therapy. The majority of the patients were on antidepressant medication and / or analgesics.

\section{Reactions to depression}

Generally the patients and their families believed that the depression would go away. The older patients were more likely to understand their depression as a mode of communication with the ancestors, and responded by consulting traditional healers to help them perform the necessary rituals. More than $40 \%$ of the patients consulted traditional healers prior to seeing a primary health care practitioner and continued to use both traditional and western forms of treatment.

\section{Reactions to health care providers}

Participants also expressed a difference in their perception of the health practitioners in traditional and western contexts. When a person who is ill seeks a traditional doctor, there is no actual consultation charge. A 'small fee/ donation' or gift is given for the doctor to 'open his bag'. The traditional doctor is only expected to be compensated when health is restored to his clients - who are often taken into his home for an extended period. This as opposed to western doctors who are paid for a consultation. This difference in practice, participants felt, makes help-seeking inaccessible and contributes to suspicion, fear and mistrust of the mental health institutions and mental health practitioners.

\section{Discussion}

This study supports previous research in showing a gender difference in help-seeking. More women presented with depression than men for the period of the audit. The ratio of women to men attending being 4:1. This gender difference has been observed for years using different measures of depression and across a variety of cultures. ${ }^{4}$ These results emphasise the need for clinical services and the use of intervention strategies to cater for the needs of its consumers. The lack of resources for women in South Africa hinders and prevents early treatment and access. Maternal health significantly impacts on the health of family and children. There is a well established correlation between mothers who are depressed and depression in their children. ${ }^{10}$ Improving women's health and accessibility to health care will appreciably impact on the general health status of families and communities.

While there was no significant difference in the mean age of either gender in this study, it has been found that the age of onset of depression is usually earlier in women than in men. ${ }^{7}$ Early helpseeking behaviour among women in this study may have been prohibited by other barriers of access such as time, financial costs, child minding, availability of sick leave and the risk of loss of employment. It has also been found that in women the duration of episodes are longer, the course of the illness more recurrent, the seasonal effect on mood greater and the association of stressful life events more frequent when compared with men. ${ }^{\text {? }}$ 


\section{Depression as a result of social stress/ impoverishment}

The finding that most of the patients with depression were single and unemployed echoes previous research conducted in the rural areas of South Africa where 'single' marital status, unemployment and low income were associated with a high prevalence of depression. ${ }^{11}$ Without employment, it is suggested that women rely on their role of housewife for identity and self-esteem which carries many frustrating elements. In modern society being a housewife is looked-down-upon as compared to women who earn their own income. ${ }^{12}$ Being single also carries its own stigma and limits the individuals' access to resources. Having a stable partner provides social support which is regarded as an important buffer against ill health. However, in this study a key finding was that many women in this study reported domestic violence as a major stressor. This is consistent with a study by Cohen et al. who found that 2 out of every 3 women had experienced domestic violence during their lifetimes..$^{13}$ Domestic violence can cause serious instability in the family. Consequences of domestic violence are split families, poorer parent-child relationships and in some cases economic deprivation. But the main problem associated with domestic violence is that children are more likely to use the aggressive tactics they see their parents using while others learn to turn their anger inwards. Links between domestic violence and low levels of self-esteem have been found and this often leads to depression in parents. ${ }^{13}$

Most patients identified 'stress' as the cause for depression, with a few patients identifying the role of the ancestors, spirituality and bewitchment. These results suggest that our sample may be reflecting a shift in traditional concepts and perceptions of depression. A similar change in trends was noted in a study on schizophrenia in a South African population in Cape Town. ${ }^{14}$ Geographical background seems to influence the meaning attributed by African women to depression. Rural subjects made more associations between their depression and traditional beliefs than urban subjects. Be that as it may these results may be suggesting a trend whereby urban African women are adhering less to traditional cultural perceptions of depression in the way that their rural counterpart do. Of interest is the regularity in which the concept 'stress' and the word 'iStress' was adopted by isiZulu speakers and integrated into the language. This 'medicalisation' is likely to have occurred through increasing exposure to health services as well as the fact that depression is a socially constructed phenomenon and perceptions of the phenomenon change over time. ${ }^{14}$ This change has important implications for treatment. Clinical services and health providers should not only make resources available and access to the resources easier but provided them in such a way as to enhance this change in help-seeking behaviours. Greater awareness of depression and mental disorders through education using all available opportunities and partnerships with business can help and will reduce the stigma associated with mental illness. In addition, devolving services to the community level, educating business to be more flexible so that patients' can keep appointments and restructuring services to minimise the barriers can also be ways that this can be achieved.

Even though there is a remarkable shift in the thinking and lifestyles of African women who have been influenced by urbanization and westernization, there remain those who are still ambivalent. However, the likelihood of co-consulting of traditional and western healers is expected to continue and hence health practitioners must be sensitive and accommodate such trans-health seeking practices. ${ }^{9}$

A look at the diagnostic distribution in this study reflects that a large number of patients presented with a Major Depressive Disorder (MDD). This finding must alert us to the presence of higher levels of actual psychopathology in existence given the poor socioeconomic conditions, political violence, domestic violence, high rates of illiteracy and other pathogenic conditions which primarily impact upon these communities. This finding again alerts us to the severe lack of psychological services for most of the population, especially at a primary prevention level. Culturally 'influenced' help-seeking behaviour may also contribute to paying attention to especially those individuals with severe symptoms and who eventually seek 'medical' interventions. Less severe or mild depressive symptoms may be treated by traditional means and/ or cared for within the community. Swartz noted that hospital services seem to be used mainly for more serious psychiatric problems within the black community. ${ }^{15}$

Most patients lacked adequate support structures. Support is an important factor in responses to stress and the importance of a supportive environment in healing has been long recognized..$^{9,15}$ This lack of support, especially among women, leaves them vulnerable to a wide range of stressful events which range from domestic violence to political violence. The high rates of sexual abuse seem to reflect the often violent social context in the township and the country generally.

\section{Depression presenting as psychosomatic illness}

Pain and suicidal behaviour were the most common symptoms/ complaints. While there as been a significant focus on the increase of suicidal behaviour among our African community ${ }^{16}$ little is understood about pain as a symptom. Clinically this high endorsement of physiological symptoms and reports of pain to the health practitioners is well known. Presenting with pain may legitimise the help seeking in a context where there are so many other competing needs for resources and the need to see a doctor. It may symbolically represent the emotional status of the patient. However physical presentation such as pain may mislead the health practitioner and contribute to the late detection of depression and other mental illnesses, particularly at the primary care level. In South Africa it has been found that thousands of patients with mental health problems are not detected at the primary health care level. ${ }^{17}$ The education of primary care providers and health practitioners at these levels about depression and other mental illnesses is crucial for better outcomes. Moreover, historically, depression was stigmatizing and prejudiced patients and their families. This may account for why it is thus more acceptable for most people to present with somatic symptoms such as headaches, backaches, etc. Pain reported by African women embodies more than just physical pain. It is a cry for help as well as a symptom of depression.

\section{Appropriateness of the psychological services offered}

It is quite concerning that patients did not continue with psychotherapy or any treatment long enough to benefit. Patients defaulted in spite of attempts by the health provider to accommodate their needs and/ or scheduling appointments for their convenience. While barriers to health, such as costs, time, and transport play an important role, numerous complex social, moral, personal and situational beliefs affect adherence to psychotherapy. ${ }^{9}$ Generally the patients and their families believed that the depression would 'just go away'. Depression in the African community is often not a primary concern and its presentation is often 'neglected'. The majority of patients live in poverty and struggle with complex economic issues as a result of homelessness, 
domestic violence, etc. Most of their energy is spent meeting basic needs such as food, housing, and employment for themselves and their families, which take priority over depression. Given this situation the use of psychotherapy as the initial or main form of intervention must be seriously reconsidered. It would appear that social intervention and social work services may be the more appropriate need and intervention.

In order to understand these issues the mental health practitioner has to think in a more discerning manner about the provision of treatments and intervention, giving more thought to the understanding and integration of socio-cultural factors. This study also highlights the need for interventions that are needs focused and which can be effectively implemented within a single session or short term. In this regard social interventions have to be seriously considered. Poor follow-up and adherence also supports the use of medication. Making anti-depressants available at the time of diagnosis and follow up at local clinics may enhance treatment and adherence.

\section{Limitations of the study}

This study was retrospective. Secondly, the use of the reported diagnostic categories did not include checks for the reliability and validity of the diagnosis across clinical psychologists who saw the participants. Third, the sample was small for generalizations to the black South African population. Despite the limitations of this study, the information obtained can be useful in the training of health workers to respond appropriately to the social and cultural factors involved in depression.

\section{Conclusion}

This study has obvious ramifications for health service planning and effective intervention strategies given the high rates as well as symptom presentation of depression noted in the current study. Mental health care practitioners should seriously consider depression as a differential diagnosis in all patients presenting with somatic complaints.

Women living with depression have a multitude of medical, psychological and social problems. They do not live in isolation but as integral members of their communities. Although in the current study defaulting with psychotherapy appointments was common, this must not be interpreted as patients not requiring or wanting help. Perhaps this suggests that a different kind of help is needed and not what has been traditionally provided or structured along the expectation and perceptions of present mental health providers. Focus groups with such women may provide insight into the particular kind of help needed. An approach that ensures the mental health practitioner addresses not only their psychological needs but also their social needs and culture beliefs will be extremely beneficial. There is a need to rigorously challenge the psychological practices based almost exclusively on dominant western conceptualizations of mental health. Psychology's understanding of the inter and intra psychic dynamics of individuals and groups; and the conceptualisation and meaning of illness and theories of behaviour change can enhance diagnosis, devise and improve interventions and improve adherence. In the context of a transforming South Africa with scarcity of resources the need to develop appropriate and cost effective interventions is essential. It seems more advantageous to include and promote more preventive strategies in the management programmes for depression and mental illness in general. One approach will be to provide appropriate skills and coaching to community health nurses, other health professionals and to train them in crisis intervention and stress management as well.

Many African women are single, heads of households, with young dependent children and who lack actual and perceived support. It should also be remembered that they return to a social context within which depression is increasingly prevalent. Community based organisations such as churches should increasingly provide support to these women. Depression in women begins from an early age and it is essential that preventative strategies should begin at school, among children and adolescents. Even though it might be difficult to improve their social circumstances, it might be helpful to provide them with appropriate interventions to improve self-confidence and enhance self-esteem at the early stages of development that are crucial to later healthy functioning. Skill building, stress reduction, problem solving techniques, and psycho-education may be a more appropriate approaches or alternatives to consider.

\section{References}

1. Pillay BJ, Cassimjee MH. Anxiety and Depression in an Urban General Practice. Journal of Anxiety and Depression 2000;3(2):4-5.

2. Rapmund V, Moore C. Women's stories of depression: a constructivist approach. South African Journal of Psychology 2000;30(2): 20-30.

3. Kockler $M$, Heun R. Gender differences of depressive symptoms in depressed and non-depressed elderly persons. Internal. Journal of Geriatric Psychiatry 2002;17: 65-72.

4. Nolen-Hoeksema S, Keita GP.Women and depression. Psychology of Women Quarterly 2003;27:89-90.

5. McKinlay JB, McKinlay SM, Brambilla D. The relative contributions of endocrine changes and social circumstances to depression in middle aged women. Journal of Health and Social Behaviour 1987;28:345-363.

6. Mkhize D, Mayekiso TV.The prevalence of depression amongst adolescents in the Transkei. Paper presented at: 9th National Congress of the South African Association of Child and Adolescent Psychiatry, 1993; Cape Town.

7. Bhatia SC, Bhatia SK. Depression in women: diagnostic and treatment considerations. American Family Physician 1999:166-178.

8. Mazure CM, Longhurst GJ, Weis EL. Childhood sexual abuse as a risk factor for adult depression in women. Psychosocial and neurological correlates. American Journal of Psychiatry 1999;156:81 6-828.

9. Pillay BJ. A Model of Help-Seeking Behaviour for Urban Africans. South African Journal of Psychology 1996;26(1): 4-9.

10. Pillay BJ, Moosa IG. A study of depression in black children at a general hospital. IFE PsychologIA. An international journal 2000;8(1):58-74.

11. Miza I, Jenkins R. Risk factors, prevalence and treatment of anxiety and depressive disorders in Pakistan:systemic review. British Medical Journal 2004;328:794-800.

12. Piccinelli P, Wilkinson G. Gender differences in depression. British Journal of Psychiatry 2000;177:486-492.

13. Cohen M, Deamant, C., Barkan, S. Domestic violence and childhood sexual abuse in HIV infected women and women at risk for HIV. American Journal of Public Health 2000;90(4):560-565.

14. Lund C, Swartz L. Xhosa-speaking schizophrenic patients' experience of their condition: psychosis and amafufunyana. South African Journal of Psychology 1998;28(2):62-70

15. Swartz S. Culture and mental health: a Southern African View. Cape Town: Oxford; 1998.

16. Schlebusch L. Suicidal behaviour in South Africa. Durban: University of KwaZulu-Natal Press; 2005.

17. Pillay AL, Naidoo P, Lockhart MR. Psychopathology in urban and rural/peri-urban children seeking mental health care. South African Journal of Psychology 1999;29(4):178-183. 